\title{
Functional Relevance of Dual Olfactory Bulbs in Olfactory Coding
}

\author{
Praveen Kuruppath, Li Bai, and Leonardo Belluscio
}

https://doi.org/10.1523/ENEURO.0070-21.2021

Developmental Neural Plasticity Section, National Institute of Neurological Disorders and Stroke, National Institutes of Health, Bethesda, MD 20892

\begin{abstract}
Bilateral convergence of external stimuli is a common feature of vertebrate sensory systems. This convergence of inputs from the bilateral receptive fields allows higher order sensory perception, such as depth perception in the vertebrate visual system and stimulus localization in the auditory system. The functional role of such bilateral convergence in the olfactory system is unknown. To test whether each olfactory bulb (OB) contributes a separate piece of olfactory information, and whether information from the bilateral $O B$ is integrated, we synchronized the activation of OBs with blue light in mice expressing ChIEF in the olfactory sensory neurons (OSNs) and behaviorally assessed the relevance of dual OBs in olfactory perception. Our findings suggest that each $\mathrm{OB}$ contributes separate components of olfactory information, and the mice integrate the bilaterally synchronized olfactory information for olfactory identity.
\end{abstract}

Key words: channelrhodopsin-2; foot shock; light zone; olfactory bulb; safe zone

\section{Significance Statement}

Identifying an odor is the first step in olfactory coding, as it is critical for the survival of most animals. Bilateral olfactory bulbs (OBs) help rodents to localize the odor source and navigate accordingly. However, it is unclear whether the bilateral OBs play a role in determining the identity of an olfactory information. In this study, by the controlled activation of unilateral and bilateral OBs with blue light in tetO-ChIEF-Citrine mouse line, we studied the behavioral responses to the light pulse stimulation in unilateral and bilateral OB. we found that each $\mathrm{OB}$ provides distinct olfactory information and rodents integrate information from the two bulbs to identify an olfactory stimulus.

Received February 16, 2021; accepted August 4, 2021; First published August 19, 2021.

The authors declare no competing financial interests.

Author contributions: P.K. and L.Be. designed research; P.K. and L.Ba. performed research; P.K. analyzed data; P.K. wrote the paper.

This work was supported by the National Institute of Neurological Disorders and Stroke at the National Institutes of Health Intramural Program Project Number 1ZIANS003116-01 (to L.Be.).

Acknowledgements: We thank National Institutes of Health Library Editing Service and Nathan E. Schoppa, University of Colorado School of Medicine, for editing the manuscript.

Correspondence should be addressed to Praveen Kuruppath at praveen. kuruppath@cuanschutz.edu.

https://doi.org/10.1523/ENEURO.0070-21.2021

Copyright (C) 2021 Kuruppath et al.

This is an open-access article distributed under the terms of the Creative Commons Attribution 4.0 International license, which permits unrestricted use, distribution and reproduction in any medium provided that the original work is properly attributed.

\section{Introduction}

The perception of odor is crucial for the survival of most animals, because it is essential for navigation, finding food sources, and avoiding predators. Convergence of sensory inputs from a bilateral receptive field is a fundamental aspect of the biological sensory systems to extract information about the environment. In the visual, auditory, and somatosensory system, this bilateral convergence is well established for depth perception (Ohzawa et al., 1997; Anzai et al., 2011), sound localization, (King et al., 2001; Konishi, 2003), and object localization (Shuler et al., 2001, 2002). The significance of such bilateral convergence and the functional relevance of dual olfactory bulbs (OBs) in the olfactory system remain unclear.

The identity of an odor, along with its concentration, are two fundamental aspects of olfactory information (Zhou 
and Belluscio, 2012; Storace and Cohen, 2017; Kepple et al., 2019; Parabucki et al., 2019). Animals move toward increasing odorant concentration to locate food, mates, and avoid predators by moving away following decreasing odorant concentrations. To achieve this effect, animals may rely on a comparison of odorant information sampled through the bilateral symmetric nostrils (Raman et al., 2008; Esquivelzeta Rabell et al., 2017). Previous studies have shown that rodents integrate bilateral cues from the nostrils to localize the odorant sources (Rajan et al., 2006; Catania, 2013). However, it is unknown whether bilateral olfactory inputs are integrated and perceived as a single olfactory information.

In this study, we controlled the sensory inputs to the OBs with blue light in tetO-ChIEF-Citrine mice line, where channelrhodopsin-2 is expressed in all the olfactory sensory neurons (OSNs) and studied the behavioral responses to the light pulse stimulation. We found that each OB provides separate olfactory information, and that the perception of an olfactory stimulus reflects the composite of information provided by the two OBs.

\section{Materials and Methods}

\section{Experimental animals}

All animal procedures conformed to National Institutes of Health guidelines. Mice were bred in-house and were maintained on a 12/12 $\mathrm{h}$ light/dark cycle with food and water ad libitum.

The tetO-ChIEF-Citrine line, was generated from pCAGGS-I-oChIEF- mCitrine-I-WPRE (7.7 kb; Roger Tsien, University of California, San Diego), which contains the coding sequence for mammalian-optimized ChIEF fused to the yellow fluorescent protein Citrine, at the National Institute of Mental Health Transgenic Core Facility (Bethesda, MD) as previously described (Lin et al., 2009; Cheetham et al., 2016). The OMP-tTA knock-in mouse line expressing the tetracycline transactivator protein (TTA) under the control of the OMPpromoter was a gift from Joseph Gagos. Experimental animals were OMP-tTA+/-/tetO-ChIEF-Citrine+/(OMP-ChIEF), generated by crossing heterozygous tetO-ChIEF-Citrine (tetO-ChIEF-Citrine+/-) with homozygous OMP-tTA mice (OMP-tTA-/-).

\section{Genotyping}

OMP-ChIEF pups were identified by the visualization of fluorescence in the nose and OB of PO-P2 (postnatal day0 - postnatal day2) pups under epifluorescence illumination.

\section{Animal preparations}

Data were collected from 12 OMP-ChIEF mice (eight males and four females) and four male wild-type mice. Experimental animals were prepared as described previously (Sparta et al., 2011; Ung and Arenkiel, 2012). Briefly, mice were anesthetized with an intraperitoneal injection of ketamine/xylazine mixture 100 and $10 \mathrm{mg} / \mathrm{kg}$ body weight, respectively. Each animal was fixed with a stereotactic frame with the head held in place by a bar tie to each temporal side of the skull. The animals were kept warm with hand warmers (Grabber). Surgery was started when the animal showed no movement in response to foot pinching. A craniotomy was performed above the skull over each OB. Fiber optic pins were implanted on the dorsal surface of each OB (4.3 $\mathrm{mm}$ anterior from bregma), as described previously (Sparta et al., 2011; Ung and Arenkiel, 2012; Li et al., 2014). Mice were injected with ketoprophen $(5 \mathrm{mg} / \mathrm{kg})$ immediately after the surgery. Animals were allowed to recover in their home cage for one week.

\section{Behavioral procedure and training}

Light stimulation and foot shock avoidance training

Behavioral training began after the animals recovered from the surgery (one week). Training was performed for 2 d on a "two-arms maze," modified from "Y" maze, with two equally sized open arms and one permanently closed arm. Each open arm was independently paved with an electric grid shock floor. The mice were connected to a 400- $\mu \mathrm{m}$ core-diameter optical fiber attached to a 473-nm solid-state variable-power laser (LaserGlow Technologies; Fig. 1A) and allowed to habituate in the two-arms maze, for $15 \mathrm{~min}$. The time spent in each arm of the two-arms maze, was recorded and assessed for arm preference. The preferred arm for the mice was selected as the light zone and the opposite arm served as the safe zone. If the mice did not demonstrate a preference for either arm, the light zone was randomly selected. After the habituation, mice were re-introduced to the two-arms maze, for the 10-min training session. A 10-min reinforcement training was performed every day $60 \mathrm{~min}$ before the test session to enhance learning. The training included light stimulation followed by a mild foot shock. The foot shock avoidance training is paired with either left or right $\mathrm{OB}$ stimulation. The light stimulation and the foot shock were delivered in the light zone when the mice completely entered that zone (Fig. 1B). The mice had free access to the safe zone to escape the foot shock. Light stimulation, consisting of a train of ten light pulses of 50-ms duration with an interval of $150 \mathrm{~ms}$, was externally triggered by a Master-8 timer (A.M.P.I.; Li et al., 2014; Erofeev et al., 2019; Quinlan et al., 2019). The output power of the light pulses was measured and adjusted to 20-22 mw with reference to previous studies (Lin, 2011, 2012; Sparta et al., 2011). The mild foot shock $(0.65 \mathrm{~mA}, 5 \mathrm{~s})$ generated by a stand-alone shock generator (Med Associates) was delivered $2 \mathrm{~s}$ after the light stimulation by Master-8 timer. The mice were trained to move to the safe zone when the light stimuli and the foot shock were delivered in the light zone.

\section{Light zone avoidance test}

The light zone avoidance test was performed for $2 \mathrm{~d}$. A reinforcement training was performed each day before the testing. The testing began $60 \mathrm{~min}$ after the reinforcement training. Before the testing, the electric grid shock floor was removed from the two-arms maze, so the mice did not experience foot shock during the test session. The activity of the mice in the two-arms maze, was evaluated in blocks of three trials and each trial was $15 \mathrm{~min}$. In the first trial, the mice were allowed to explore the arena without any light stimulation and assessed the baseline 
A

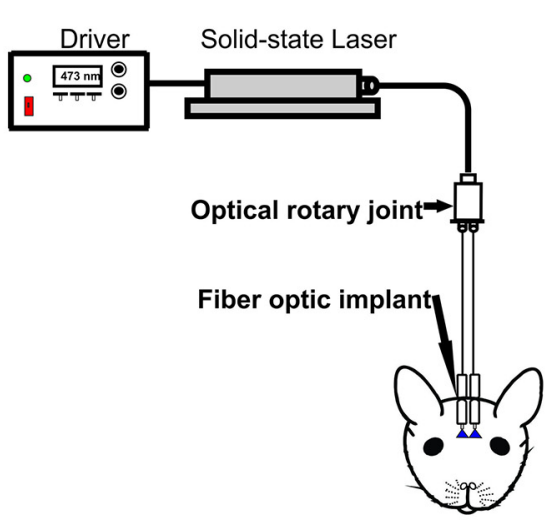

B

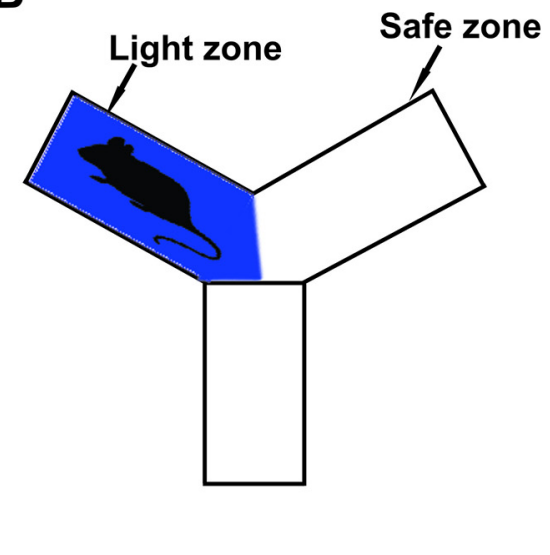

\section{C}

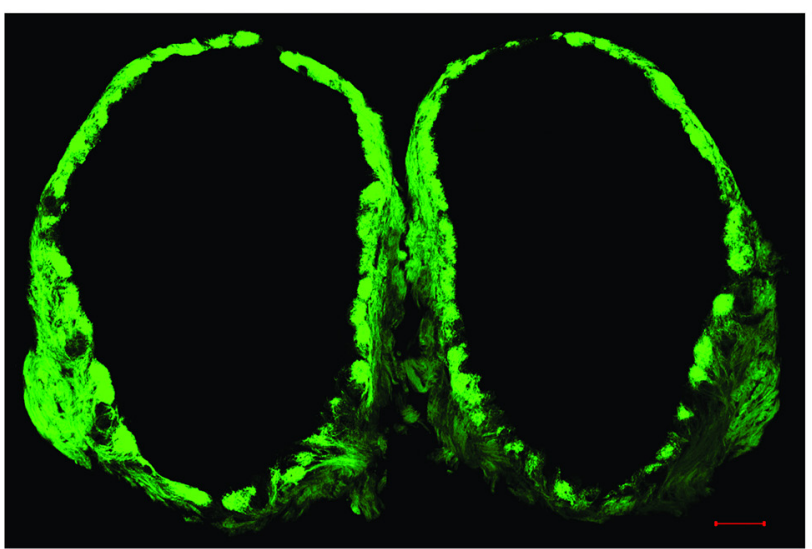

Figure 1. Optogenetic stimulation of OB. $\boldsymbol{A}$, Schematic diagram of the functional system. $\boldsymbol{B}$, Behavioral setup. Light zone indicates the area where the light stimulation is delivered. Safe zone indicates the area where mice can escape from the light stimulation and foot shock. C, Fluorescence image of the OB in an OMP-ChIEF mouse. Green indicates the expression of ChIEF tagged with a green fluorescent protein (ChIEF-GFP). Scale bar: $100 \mu \mathrm{m}$.

behavior. The time spent in each arm was calculated to evaluate arm preferences after the foot shock training session. For the following trials, the light zone and safe zone were selected, as indicated previously. The time spent in each arm was calculated by tracking the animal's movement in the two-arms maze, using Any-maze video tracking software (Stoelting). A heatmap was also generated for each trial which displays the amount of time mice spent in different parts of the arena. A range of colors indicate the total time spent in the area with blue indicating the shortest and the red as the longest time.

\section{Perfusion and immunohistochemistry}

At the end of behavioral testing, the mice were anesthetized with $200 \mathrm{mg} / \mathrm{kg}$ ketamine and $20 \mathrm{mg} / \mathrm{kg}$ xylazine and transcardially perfused with ice-cold PBS followed by $4 \%$ paraformaldehyde (PFA). OB were dissected out and embedded in $10 \%$ gelatin and postfixed/cryopreserved in $15 \%$ sucrose and $2 \%$ PFA in PBS overnight at $4^{\circ} \mathrm{C}$, cryopreserved in $30 \%$ sucrose in PBS for $24 \mathrm{~h}$ at $4^{\circ} \mathrm{C}$ and flash frozen in 2-methyl butane on dry ice. Coronal sections were cut using a cryostat (Leica Microsystems) at $50 \mu \mathrm{m}$ and stored at $-80^{\circ} \mathrm{C}$.
For immunohistochemistry, free-floating sections were incubated for $20 \mathrm{~min}$ in $1 \%$ sodium borohydride in TBS, blocked in the blocking medium containing $5 \%$ horse serum, $0.1 \%$ gelatin and $0.5 \%$ Triton $\mathrm{X}-100$ for $1 \mathrm{~h}$, and incubated with OMP primary antibody (goat, Wako, 1:1000) in $3 \%$ horse serum and $0.2 \%$ Triton $\mathrm{X}-100$ for $24 \mathrm{~h}$ at $4^{\circ} \mathrm{C}$, then donkey-anti-goat Cy3 secondary antibody (Jackson ImmunoResearch, 1:600) for $2 \mathrm{~h}$ at room temperature. Sections were mounted in Vectashield mounting medium (Vector Laboratories). Images were acquired using a Zeiss LSM 510 laser scanning confocal microscope.

\section{Statistical analysis}

All statistical analyses were done by GraphPad Prism software (GraphPad). Statistics are displayed as mean \pm SEM. Paired $t$ test was used for the comparison. Differences were determined significant when $p<0.05$.

\section{Results}

\section{Each OB provides distinct olfactory information}

Previous studies have shown that odor information is projected unilaterally (Marrone, 2013) in the vertebrate 
A

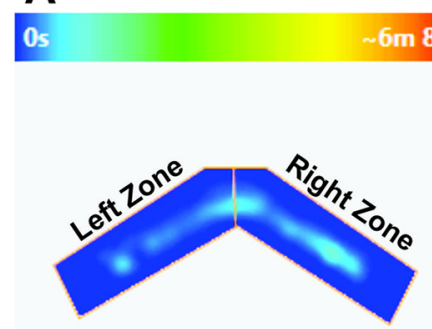

D

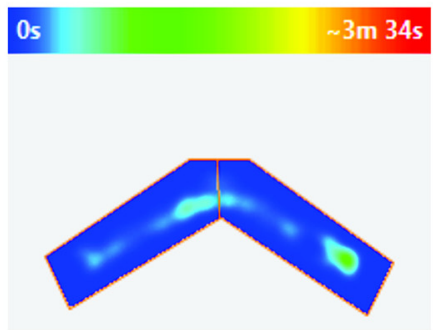

G

0s

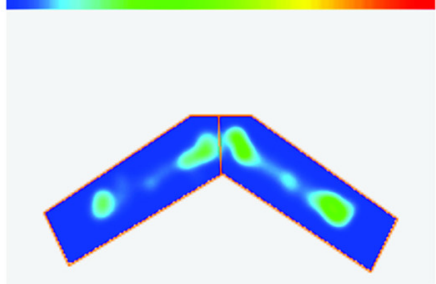

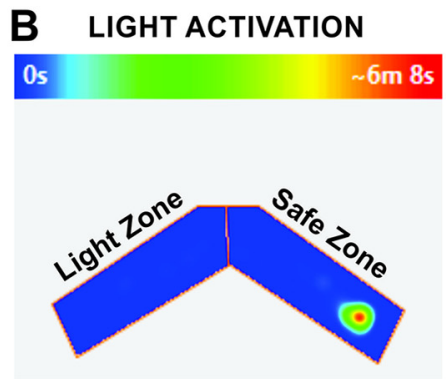

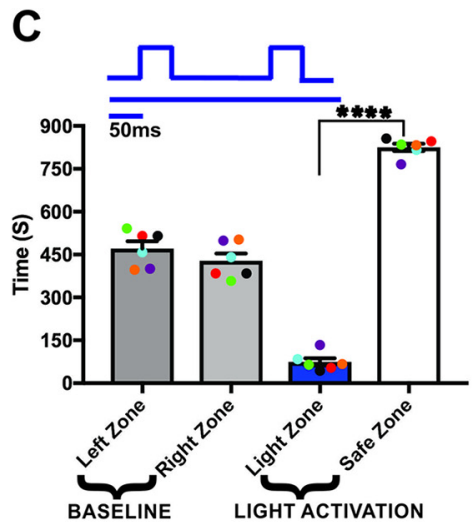

E

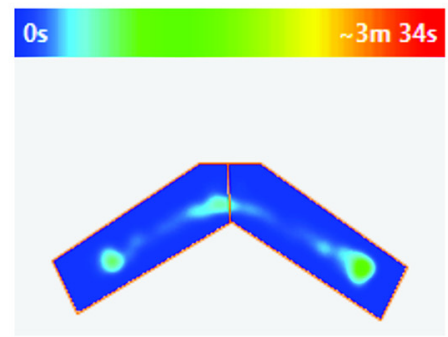

$\mathbf{F}$

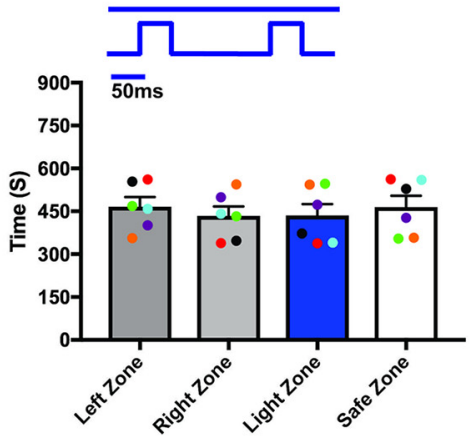

I

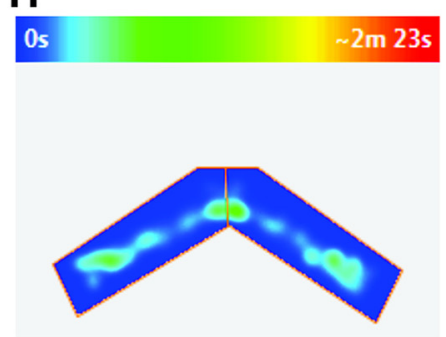

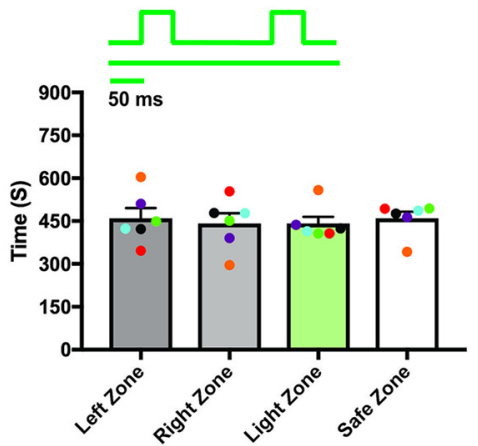

Figure 2. Distinct odor information from individual OB. $\boldsymbol{A}, \boldsymbol{B}$, Example of heat-map showing animal's position in two-arms maze, during baseline $(\boldsymbol{A})$ and ipsilateral OB stimulation $(\boldsymbol{B})$. $\boldsymbol{C}$, Average amount of time explored in each zone in baseline (left zone, right zone) and ipsilateral OB stimulation trials (light zone, safe zone). $\boldsymbol{D}, \boldsymbol{E}$, Heat-map of mouse position during baseline (D) and contralateral $\mathrm{OB}$ stimulation $(\boldsymbol{E})$. $\boldsymbol{F}$, Average amount of time spent in each zone in baseline and contralateral OB stimulation trials. $\mathbf{G}, \boldsymbol{H}$, Heat-map of mouse position during baseline $(\boldsymbol{G})$ and ipsilateral green light stimulation $(\boldsymbol{H})$. $\boldsymbol{I}$, Average amount of time spent in each zone in ipsilateral green light stimulation $\left(^{* * * *} p<0.0001, n=6\right.$ animals).

olfactory system, indicating that odors may be encoded separately in each hemisphere. To investigate the unilateral coding of olfactory information, we optically stimulated ChIEF expressing OSNs in each OB (Fig. 1C) and assessed the behavioral response to light stimulation. Here, we performed foot shock avoidance training paired with either left or right OB stimulation. To avoid the activation of same glomeruli in each $\mathrm{OB}$, we implanted the optical fiber in different locations in each OB. Therefore, the light stimulation on each $\mathrm{OB}$ activates a different set of glomeruli, eliciting different olfactory information in each OB. After training, we tested the light zone avoidance response to both ipsilateral and contralateral OB stimulation. First, as a control, we calculated the time spent in each arm by allowing the mice to freely explore the arena in the absence of stimulation. The aim of the baseline behavior analysis was to determine whether the mice have any preference to a particular arm of the two-arms maze, after the foot shock training. Our baseline data show that mice spent equal amounts of time in both arms of the 


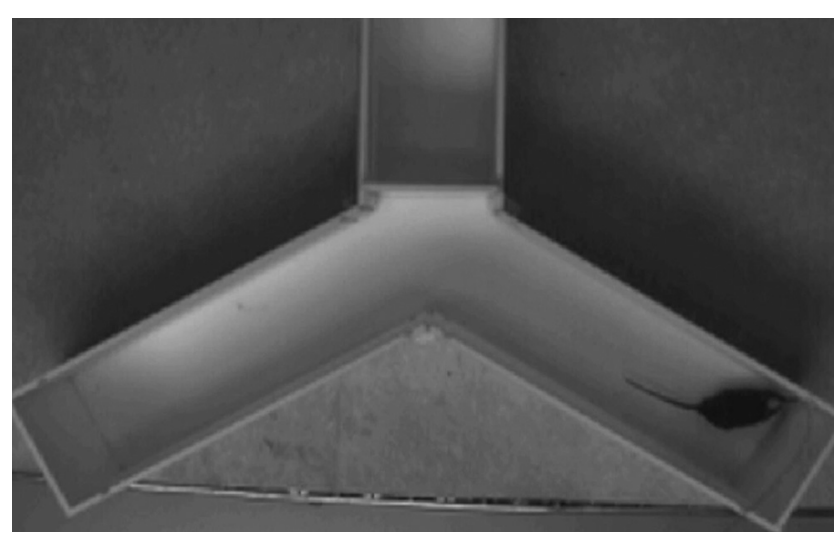

Movie 1. Unilateral foot shock trained mice avoid light zone during ipsilateral OB stimulation. [View online]

maze (left zone, $471.6 \pm 25.57 \mathrm{~s}$, right zone, $428.4 \pm$ $25.57 \mathrm{~s}, p=0.44$; Fig. $2 A$ ). Then, we delivered the light stimulation to ipsilateral $\mathrm{OB}$. We found that, mice avoided the light zone during the ipsilateral OB stimulation and spent most of their time in the safe zone (light zone, $74.67 \pm 13.07 \mathrm{~s}$; safe zone, $825.3 \pm$ $13.07 \mathrm{~s}, p \leq 0.0001$; Fig. 2B,C; Movie 1, $n=6$ ). We also tested whether the avoidance response to the light stimulation was equally probable in both arms of the maze by delivering the stimulation when the mice reached the safe zone. We found that mice avoided the safe zone during the ipsilateral light stimulation, indicating that the avoidance response is clearly linked to the olfactory information, but not to spatial information. Since we did not see a difference in the avoidance response, we pooled the data from both stimulus groups.

Next, we performed light stimulation on the contralateral OB. Our results show that the time spent in each arm during the baseline behavior trial (left zone, $466.4 \pm 33.25 \mathrm{~s}$, right zone, $433.6 \pm 33.25 \mathrm{~s}, p=0.64$; Fig. $2 D$ ) and the light activation trial (light zone, $435.3 \pm 39.93 \mathrm{~s}$, safe zone, $464.7 \pm 39.93 \mathrm{~s}, p=0.73$; Fig. $2 E$ ) were similar, indicating that the mice did not identify the foot-shock linked olfactory information. Therefore, they did not avoid the light

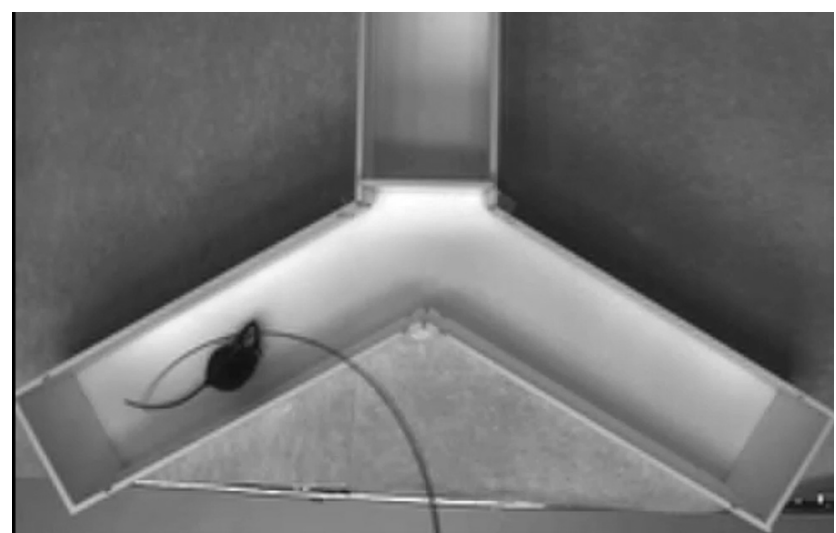

Movie 2. Unilateral foot shock trained mice did not avoid light zone during contralateral OB stimulation. [View online] zone during contralateral OB stimulation (Fig. 2F; Movie 2). This suggests that each $\mathrm{OB}$ can deliver separate light-induced olfactory information that can be discriminated by the mice.

To verify that the observed responses from the light stimulation were the result of activation of the ChIEFexpressing neurons and not from the use of light as a visual cue, we used green light $(540 \mathrm{~nm}$, output power, 20-22 mw), which does not activate ChIEF. Mice are much less sensitive to such long wavelength light (Denman et al., 2017, 2018; Peirson et al., 2018). During the green light stimulation, we did not observe significant behavioral difference from the baseline behavior (left zone, $459 \pm 36.13 \mathrm{~s}$ and right zone, $441 \pm$ $36.13 \mathrm{~s}, p=0.81$; light zone, $441 \pm 23.88 \mathrm{~s}$ and safe zone, $459 \pm 23.88 \mathrm{~s}, p=0.72$; Fig. $2 \mathrm{G}-l)$, confirming that the mice did not use visual cues to perform the task. We also performed the test with wild-type mice and confirmed that visual cues were not involved in solving the behavioral task [left zone, $457.8 \pm 40.21 \mathrm{~s}$ and right zone, $442.3 \pm 40.21 \mathrm{~s}, p=0.86$; light zone, $451 \pm 18.03 \mathrm{~s}$ and safe zone, $449 \pm 18.03 \mathrm{~s}, p=0.96$ (Fig. $3 A$ ); left zone, $445.8 \pm 32.33 \mathrm{~s}$ and right zone, $454.3 \pm 32.33 \mathrm{~s}, p=0.90$; light zone, $458.8 \pm 27.30 \mathrm{~s}$ and safe zone, $441.3 \pm 27.30 \mathrm{~s}, p=0.77$ (Fig. $3 B$, $n=4)]$. These results indicate that OMP-ChIEF mice were using only the light activation of the olfactory system as the cue to solve the behavioral task, rather than light detection via other modalities.

\section{Bilateral integration of olfactory information}

Previous tracer injection studies have shown that olfactory information is exchanged to the contralateral $\mathrm{OB}$ through anterior olfactory nucleus pars externa (AONpE; Yan et al., 2008). However, it is unknown whether the olfactory information from both OBs is integrated in odor perception. To assess whether the bilateral olfactory information is integrated in olfactory perception, we delivered synchronized light stimulation to both OBs of unilaterally trained mice and observed if the mice can distinguish the foot-shock linked olfactory stimulus from synchronized dual bulb stimulation. We found that mice visited both arms for similar amounts of time during the baseline behavior trials versus trials in which the two OBs were synchronously stimulated (left zone, $438.2 \pm 20.67 \mathrm{~s}$, right zone, $461.8 \pm 20.67 \mathrm{~s}$, $p=0.59$; light zone, $447.8 \pm 39.57 \mathrm{~s}$, safe zone, 452.2 $\pm 39.57 \mathrm{~s}, p=0.95$; Fig. $4 A$; Movie 3 ). This implies that mice detected the synchronized bilateral olfactory stimulus differently from the unilateral olfactory stimulus linked to the foot-shock. Thus, mice perceive the combined olfactory information received from the ipsilateral and contralateral OBs during synchronized bilateral $\mathrm{OB}$ stimulation differently from the information received from each bulb.

To verify that there is bilateral integration, we delivered the light stimulation asynchronously with a $50-\mathrm{ms}$ delay between ipsilateral and contralateral $\mathrm{OB}$ stimulation. This protocol assumed that asynchronous $\mathrm{OB}$ stimulation did not allow the mice to integrate the olfactory information 
A

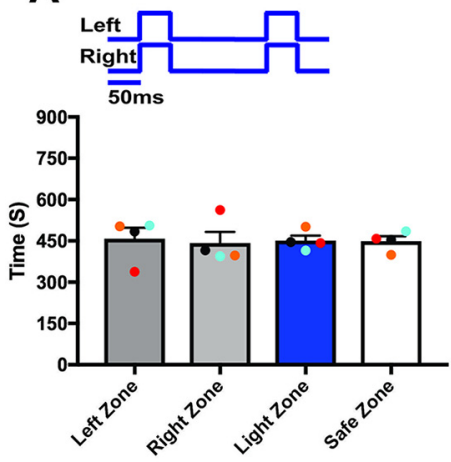

B

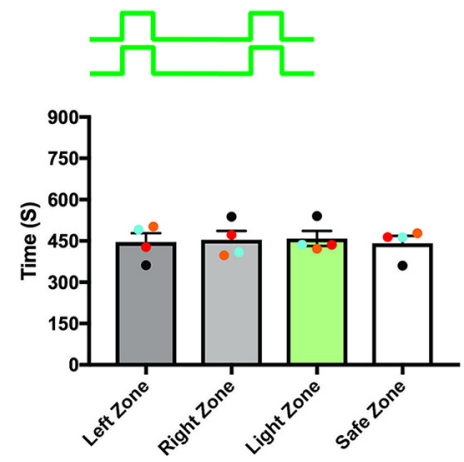

Figure 3. Light stimulation did not activate the olfactory system of the wild-type mice. A, Average amount of time spent in each zone in baseline and experiment trials during blue light stimulation. $\boldsymbol{B}$, Average amount of time spent in each zone in baseline and experiment trials during green light stimulation $(n=4)$.

because each $O B$ receives the light stimulation at a different time. We observed that when the OBs were stimulated asynchronously, mice identified a distinct light-driven olfactory stimulus paired with the footshock and avoided the light zone (light zone, $77.58 \pm$ $13.36 \mathrm{~s}$; safe zone, $822.4 \pm 13.36 \mathrm{~s}, p \leq 0.0001$; Fig. $4 B$; Movie 4). We also evaluated the difference in the avoidance response when the contralateral $\mathrm{OB}$ received light stimulation before the ipsilateral OB. This was done by, in some of the trials, first stimulating the contralateral OB before the ipsilateral OB and examining whether the mice continued to avoid the light zone. We found that mice were able to identify the olfactory stimulus paired with the foot-shock and avoided the light zone. Since we did not see a difference in the avoidance response, we pooled the data from both stimulus groups. Together, these results suggest that during asynchronous stimulation, olfactory information from the two OBs is processed separately.

\section{Unified perception of bilateral olfactory inputs}

Sensory information from bilateral receptive fields is combined into a single, unified percept in most sensory systems. However, the presence of a unified perception in the vertebrate olfactory system is unknown. To determine the unified perception of bilateral olfactory stimulation, we performed light stimulation and foot-shock avoidance training on a new group of animals $(n=6)$. Here, we delivered light stimulation to both OBs simultaneously and paired it with foot shock. After the training, we tested the response of mice in the light zone avoidance test during synchronized bilateral OB stimulation. Our results show that when both OBs were synchronously stimulated, mice avoided the light zone, indicating that mice can identify the olfactory information linked to the foot-shock (light zone, $83.67 \pm 8.891 \mathrm{~s}$; safe zone, $816.3 \pm 8.891 \mathrm{~s}, p \leq$ 0.0001 ; Fig. $5 A$; Movie $5, n=6$ ). We then tested whether the mice identify the foot-shock linked olfactory stimulus when the two OBs are stimulated asynchronously. This protocol assumes that if the mice relied on the olfactory information from the left or right $\mathrm{OB}$ to avoid the light zone, mice would identify the foot-shock linked olfactory stimulus and avoid the light zone. In contrast to this prediction, we found that the mice did not identify the olfactory information linked with foot-shock and continued to stay in the light zone (light zone, $450.2 \pm 42 \mathrm{~s}$; safe zone, $449.8 \pm 42 \mathrm{~s}$, $p=0.99$; Fig. 5B; Movie 6). Our results suggest that the mice integrate the olfactory information from both OBs during synchronized stimulation and perceive it as one olfactory stimulus. However, during the asynchronized stimulation, mice perceived two independent streams of information.
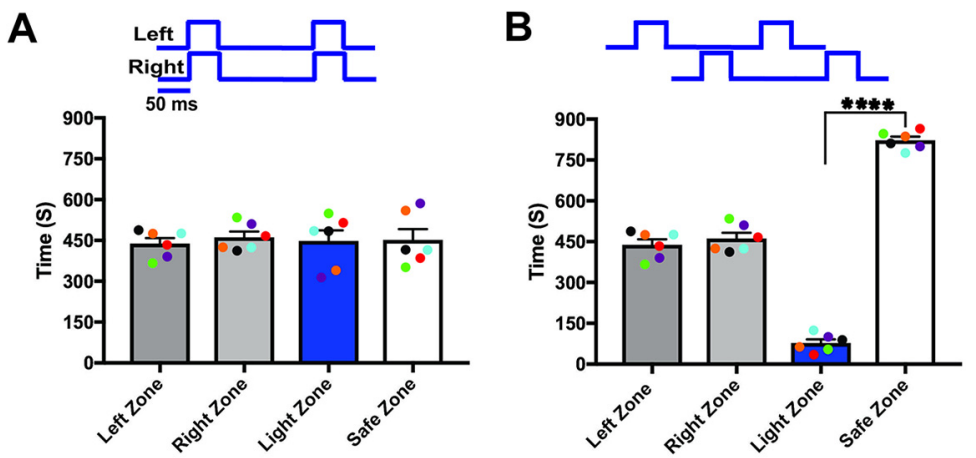

Figure 4. Synchronized bilateral OB stimulation alters the unilateral odor identity. $\boldsymbol{A}, \boldsymbol{B}$, Mean time explored during the synchronous and asynchronous OB stimulation to the unilaterally trained mice ${ }^{\star \star \star \star} p<0.0001, n=6$ animals). 


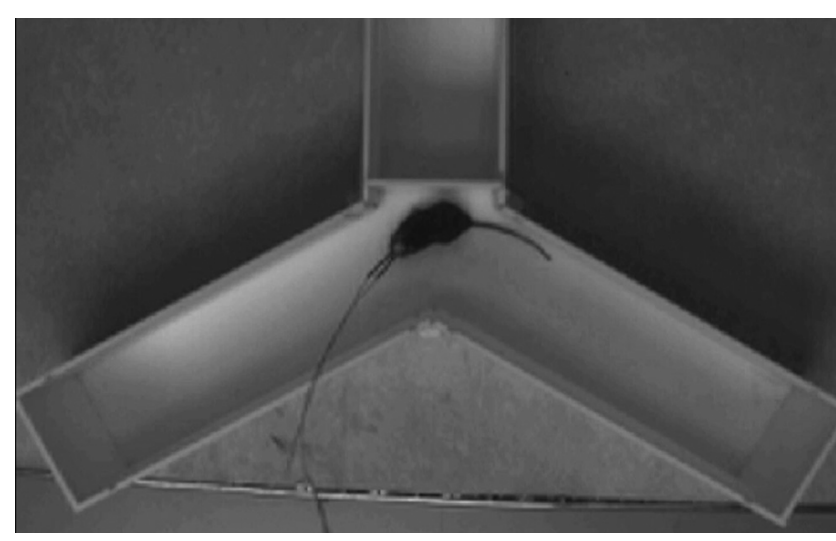

Movie 3. Unilateral foot shock trained mice did not avoid light zone during synchronized bilateral OB stimulation. [View online]

Next, we tested whether the mice can identify the footshock linked bilateral olfactory stimulus from single OB stimulation. We independently stimulated the left and right OBs of the bilaterally trained mice. We found that during the independent activation of each OB, mice did not avoid the light zone, but spent equal or additional time in the light zone as in baseline behavior trials [left zone, $425.5 \pm 29.7 \mathrm{~s}$ and right zone, $474.5 \pm 29.7 \mathrm{~s}, p=0.45$; light zone, $489.3 \pm 43.26 \mathrm{~s}$ and safe zone, $410.7 \pm 43.26$ s, $p=0.40$ (Fig. $5 C$ ); left zone, $430.3 \pm 25.72 \mathrm{~s}$ and right zone, $469.7 \pm 25.72 \mathrm{~s}, p=0.48$; light zone, $545 \pm 39.18 \mathrm{~s}$ and safe zone, $355 \pm 39.18 \mathrm{~s}, p=0.06$ (Fig. $5 D$ )]. This suggests that unilateral stimulation of each $\mathrm{OB}$ provides only a portion of the information delivered by bilateral olfactory stimulation. The result confirms our previous hypothesis that the two OB provide two separate pieces of information, which are encoded independently in each hemisphere. Furthermore, our results demonstrate that each OB provide distinct olfactory information, which can be combined and perceived as a single olfactory stimulus.

Finally, we visually compared the OMP positive OSNs in 12-week-old OMP-ChIEF control, experimental and wildtype mice and examined whether light stimulation induced a reduction in ChIEF expression and degeneration

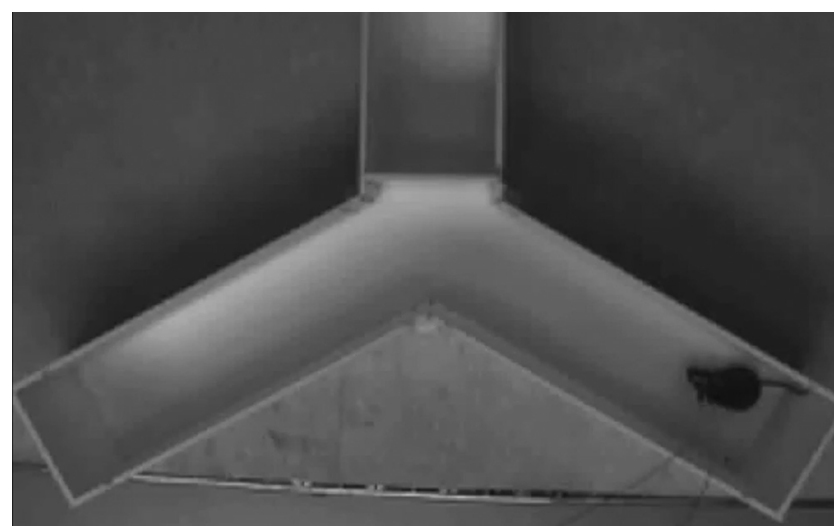

Movie 4. Unilateral foot shock trained mice avoid light zone during asynchronous bilateral OB stimulation. [View online] of OMP positive neurons in the wild-type and experimental mice. Our immunohistochemistry results show that light stimulation did not induce a reduction in the ChIEF expression or degeneration of the OMP positive OSNs (Fig. 6A-C).

\section{Discussion}

Several studies have shown that rodents use bilateral cues to localize odor source for navigation, finding food sources, and avoiding danger. Balanced and lateralized sensory inputs are the key strategies typically involved in bilateral localization. Recent studies in rodents show that odor discrimination require lateralization of the olfactory information, demonstrating the significance of lateralized sensory inputs in odor discrimination (Cohen et al., 2015; Cohen and Wilson, 2017). A previous study in humans by Thuerauf and colleagues also demonstrated the significance of lateralization for the perception of odor intensity and hedonic evaluation (pleasantness/unpleasantness; Thuerauf et al., 2008). Their study show that olfactory intensity estimates represent the most sensitive parameter of olfactory lateralization, suggesting that olfactory laterality is mainly based on intensity rather than identity.

While these studies report the significance of bilateral sampling for accurate odor source localization (Porter et al., 2005; Rajan et al., 2006; Louis et al., 2008; Catania, 2013; Esquivelzeta Rabell et al., 2017), none of the studies examined the significance of bilateral integration of olfactory information for identifying an odor. Taking advantage of the transgenic mice, our study showed that rodents combine the synchronously sampled olfactory information provided by the two OBs into a single olfactory percept. The integration of such bilateral olfactory information is likely to play a central role in the exchange of olfactory information and the formation of a unified odor percept may help to generate behaviorally relevant information regarding the animal's surrounding environment.

A previous study reported that olfactory information is projected unilaterally (Shepherd, 2004), suggesting that odorant information is encoded separately in each hemisphere. Consistent with this, our study provides evidence for distinct olfactory information processing in each hemisphere. We show that, when stimulated independently, the ipsilateral and contralateral OBS provide unique olfactory information, and mice can clearly identify the olfactory information from two bulbs (Fig. 2C,F).

In humans, Zhou and Chen (Zhou and Chen, 2009) examined how odors are perceived when each nostril receives a different odor. In their experiment, phenylethyl alcohol (PEA; rose like smell) and n-butanol (marker like smell) were delivered to each nostril simultaneously and the subjects were asked to report whether the odor perceived was "rose" or "marker." They found that the subjects perceived either the marker or the rose smell. The odor perception was constant within a sniff and could change between sniffs. This suggests that although each hemisphere presumably perceived a different odor, only one percept dominated rather than both contributing 
A

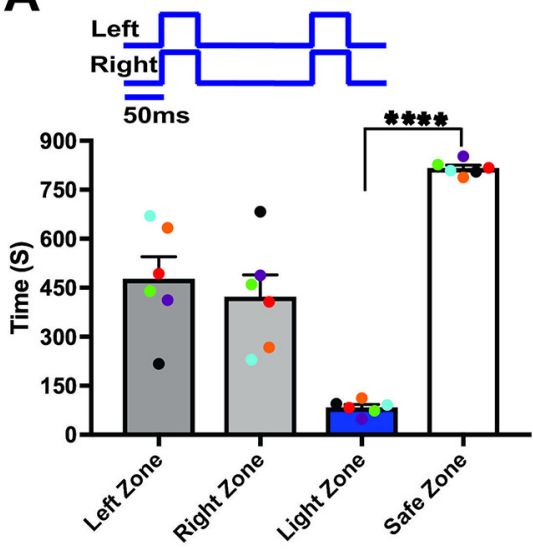

C

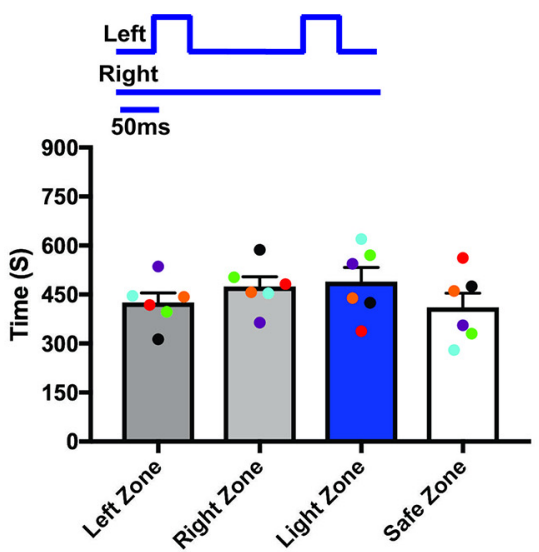

B
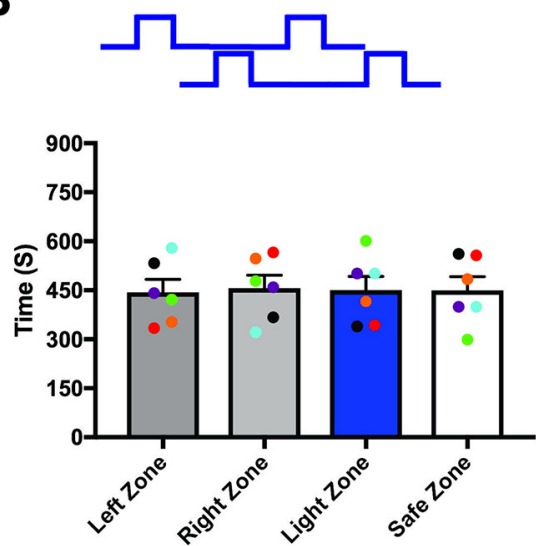

D

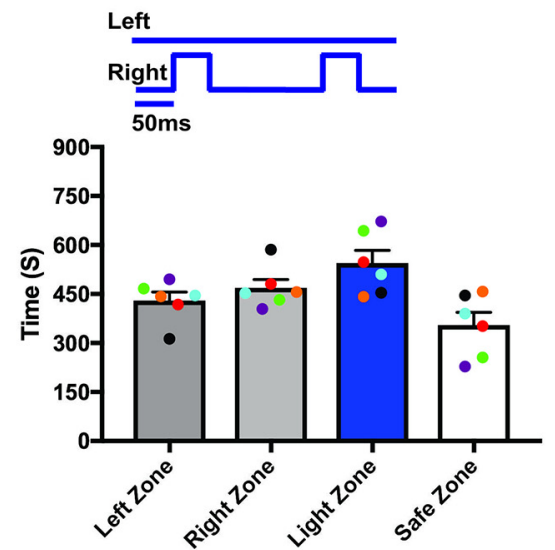

Figure 5. Unified perception of synchronized bilateral olfactory inputs. $\boldsymbol{A}, \boldsymbol{B}$, Mean time explored during the synchronized and asynchronized bilateral $\mathrm{OB}$ stimulation to the bilaterally trained mice. $\boldsymbol{C}, \boldsymbol{D}$, Mean time explored during unilateral light stimulation ${ }^{* * \star *} p<0.0001, n=6$ animals).

equally. Sniffing, flow rate and the complex interactions between odorants and the epithelium may influence this dominance of one percept leading to the perception of one odorant than contributing equally from each $\mathrm{OB}$.

The functional relevance of the dual OBs in olfactory information processing is still an open question. A recent

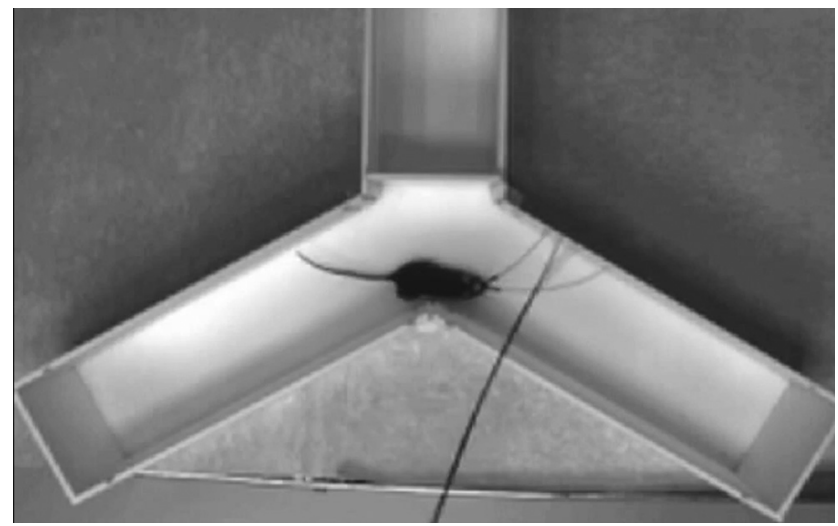

Movie 5. Bilateral foot shock trained mice avoid light zone during synchronized bilateral OB stimulation. [View online] study in Drosophila, found that olfactory receptor neurons (ORNs) project bilaterally to both sides of the brain. When an odor activates the antennal lobe asymmetrically, ipsilateral central neurons begin to spike a few milliseconds earlier at a higher rate than the contralateral neurons,

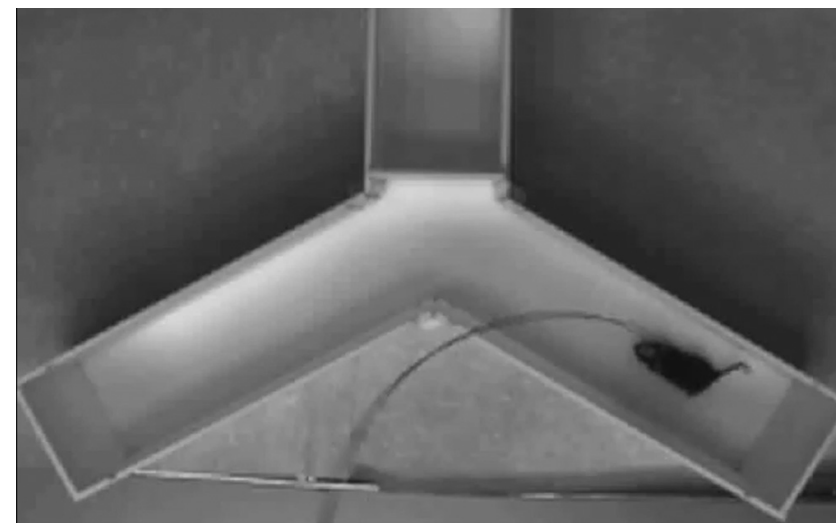

Movie 6. Bilateral foot shock trained mice did not avoid light zone during asynchronous bilateral OB stimulation. [View online] 

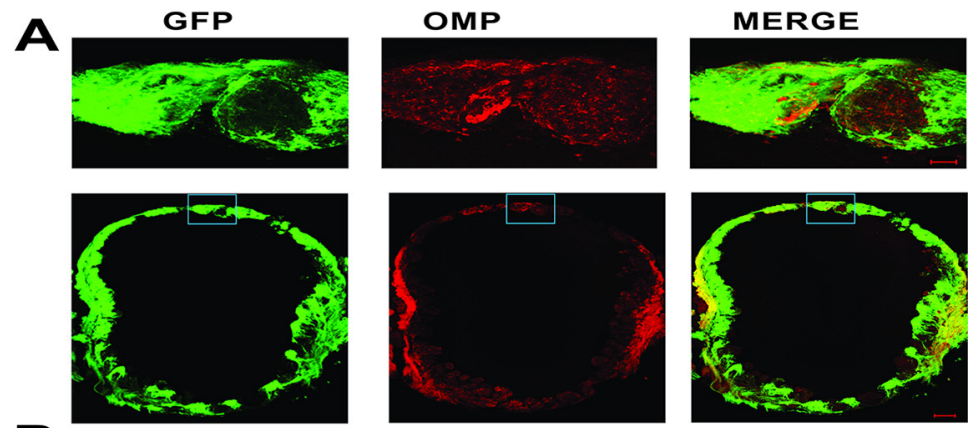

$\mathbf{B}$
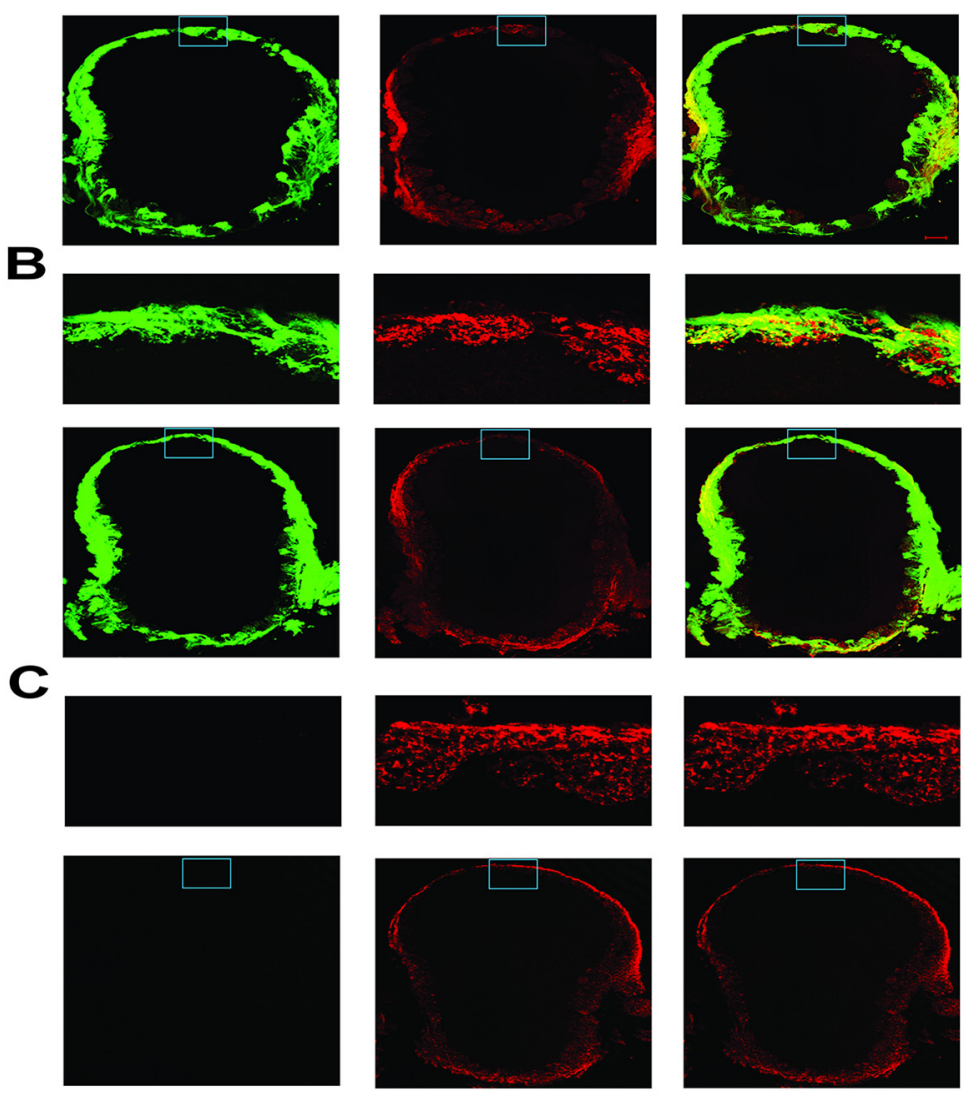

Figure 6. ChIEF expression in OMP (Olfactory marker protein) positive neurons. A, ChIEF expression in OMP positive OSNs in 12week-old OMP-ChIEF control animal. $\boldsymbol{B}$, ChIEF expression in OMP positive OSNs in 12-week-old OMP-ChIEF experimental animal. $\boldsymbol{C}$, No ChIEF expression on the OMP positive neurons of 12-week-old wild-type animal. Scale bars: $100 \mu \mathrm{m}(\boldsymbol{A}-\boldsymbol{C})$ and $20 \mu \mathrm{m}$ (inset).

allowing the fly to localize the direction of the odor source (Gaudry et al., 2013).

Previous studies in rodents reported that isofunctional glomeruli are interconnected not only within the hemisphere, but also between the hemispheres through AON, creating a mirror symmetric olfactory map in each hemisphere (Schoenfeld and Macrides, 1984; Ressler et al., 1994; Mombaerts et al., 1996; Wilson, 1997; Uchida et al., 2000; Mainland et al., 2002; Grobman et al., 2018; Kermen et al., 2020). Together, these reports suggest that dual OBs enhance the odor source localization and navigation. However, Grobman and coworkers (Grobman et al., 2018) suggest that bilateral projection is not necessary to get a bilateral response difference. Instead, a unilateral projection can result in a strong bilateral response difference. Their study shows that a unilateral projection pathway generates a higher bilateral response difference than the bilateral pathway. Since response strength is correlated with response latency, the time difference in response latency will be higher in unilateral pathway than the bilateral pathway, achieving a better odor localization in unilateral pathway. Their study suggest that the main role of bilateral projection is not odor localization, but the sharing of odor identity information across the hemispheres.

In agreement with this study, we propose that light stimulation of a single OB forms a neural representation of an odor's molecular identity in both hemispheres. In addition, we propose that the final odor identity is represented not only by the combinatorial activation of specific ORs, as previously reported (Malnic et al., 1999; Saito et al., 2009), but also by the integration of the olfactory information from the contralateral $\mathrm{OB}$. Based on the previous study (Grobman et al., 2018) and our results, we suggest that one of the main roles of dual OB is the coding of odor identity. Further research is required to fully understand how olfactory cortical neurons integrate the dual olfactory information for odor identity and odor localization and the significance of the bilateral integration of olfactory information.

In natural environments, animals confront more complex problems such as, having to identify the quality, and complexity of an odor mixture. The use of suitable 
physiological and psychophysical paradigms will be a crucial step for further understanding the complexity of neural coding in the olfactory system.

\section{References}

Anzai A, Chowdhury SA, DeAngelis GC (2011) Coding of stereoscopic depth information in visual areas V3 and V3A. J Neurosci 31:10270-10282.

Catania KC (2013) Stereo and serial sniffing guide navigation to an odour source in a mammal. Nat Commun 4:1441.

Cheetham CEJ, Park U, Belluscio L (2016) Rapid and continuous activity-dependent plasticity of olfactory sensory input. Nat Commun 7:10729.

Cohen Y, Wilson DA (2017) Task-correlated cortical asymmetry and intra- and inter-hemispheric separation. Sci Rep 7:14602.

Cohen Y, Putrino D, Wilson DA (2015) Dynamic cortical lateralization during olfactory discrimination learning. J Physiol 593:1701-1714.

Denman D, Luviano J, Ollerenshaw D, Cross S, Williams D, Buice M, Olsen S, Reid RC (2017) Mouse hue and wavelength-specific luminance contrast sensitivity are non-uniform across visual space. bioRxiv 174631 .

Denman DJ, Luviano JA, Ollerenshaw DR, Cross S, Williams D, Buice MA, Olsen SR, Reid RC (2018) Mouse color and wavelength-specific luminance contrast sensitivity are non-uniform across visual space. Elife 7:e31209.

Erofeev A, Gerasimov E, Lavrova A, Bolshakova A, Postnikov E, Bezprozvanny I, Vlasova OL (2019) Light stimulation parameters determine neuron dynamic characteristics. Appl Sci 9:3673.

Esquivelzeta Rabell J, Mutlu K, Noutel J, Martin del Olmo P, Haesler S (2017) Spontaneous rapid odor source localization behavior requires interhemispheric communication. Curr Biol 27:1542-1548.e4.

Gaudry Q, Hong EJ, Kain J, De Bivort BL, Wilson RI (2013) Asymmetric neurotransmitter release enables rapid odour lateralization in Drosophila. Nature 493:424-428.

Grobman M, Dalal T, Lavian H, Shmuel R, Belelovsky K, Xu F, Korngreen A, Haddad R (2018) A mirror-symmetric excitatory link coordinates odor maps across olfactory bulbs and enables odor perceptual unity. Neuron 99:800-813.e6.

Kepple DR, Giaffar H, Rinberg D, Koulakov AA (2019) Deconstructing odorant identity via primacy in dual networks. Neural Comput 31:710-737.

Kermen F, Lal P, Faturos NG, Yaksi E (2020) Interhemispheric connections between olfactory bulbs improve odor detection. PLoS Biol 18:e3000701.

King AJ, Schnupp JWH, Doubell TP (2001) The shape of ears to come: dynamic coding of auditory space. Trends Cogn Sci 5:261270.

Konishi M (2003) Coding of auditory space. Annu Rev Neurosci 26:31-55.

Li A, Gire DH, Bozza T, Restrepo D (2014) Precise detection of direct glomerular input duration by the olfactory bulb. J Neurosci 34:16058-16064.

Lin JY (2011) A user's guide to channelrhodopsin variants: features, limitations and future developments. Exp Physiol 96:19-25.

Lin JY (2012) Optogenetic excitation of neurons with channelrhodopsins: light instrumentation, expression systems, and channelrhodopsin variants. Prog Brain Res 196:29-47.

Lin JY, Lin MZ, Steinbach P, Tsien RY (2009) Characterization of engineered channelrhodopsin variants with improved properties and kinetics. Biophys J 96:1803-1814.

Louis M, Huber T, Benton R, Sakmar TP, Vosshall LB (2008) Bilateral olfactory sensory input enhances chemotaxis behavior. Nat Neurosci 11:187-199.

Mainland JD, Bremner EA, Young N, Johnson BN, Khan RM, Bensafi M, Sobel N (2002) Olfactory plasticity: one nostril knows what the other learns. Nature 419:802.
Malnic B, Hirono J, Sato T, Buck LB (1999) Combinatorial receptor codes for odors. Cell 96:713-723.

Marrone P (2013) Chambers, RT. Etica e Politica 15:583-605.

Mombaerts P, Wang F, Dulac C, Chao SK, Nemes A, Mendelsohn M, Edmondson J, Axel R (1996) Visualizing an olfactory sensory map. Cell 87:675-686.

Ohzawa I, DeAngelis GC, Freeman RD (1997) The neural coding of stereoscopic depth. Neuroreport 8:iii-xii.

Parabucki A, Bizer A, Morris G, Munoz AE, Bala ADS, Smear M, Shusterman R (2019) Odor concentration change coding in the olfactory bulb. eNeuro 6:ENEURO.0396-18.2019.

Peirson SN, Brown LA, Pothecary CA, Benson LA, Fisk AS (2018) Light and the laboratory mouse. J Neurosci Methods 300:26-36.

Porter J, Anand T, Johnson B, Khan RM, Sobel N (2005) Brain mechanisms for extracting spatial information from smell. Neuron 47:581-592.

Quinlan MAL, Strong VM, Skinner DM, Martin GM, Harley CW, Walling SG (2019) Locus coeruleus optogenetic light activation induces long-term potentiation of perforant path population spike amplitude in rat dentate gyrus. Front Syst Neurosci 12:67.

Rajan R, Clement JP, Bhalla US (2006) Rats smell in stereo. Science 311:666-670

Raman B, Ito I, Stopfer M (2008) Bilateral olfaction: two is better than one for navigation. Genome Biol 9:212.

Ressler KJ, Sullivan SL, Buck LB (1994) Information coding in the olfactory system: evidence for a stereotyped and highly organized epitope map in the olfactory bulb. Cell 79:1245-1255.

Saito H, Chi Q, Zhuang H, Matsunami H, Mainland JD (2009) Odor coding by a mammalian receptor repertoire. Sci Signal 2:ra9.

Schoenfeld TA, Macrides F (1984) Topographic organization of connections between the main olfactory bulb and pars externa of the anterior olfactory nucleus in the hamster. J Comp Neurol 227:121-135.

Shepherd GM (2004) The synaptic organization of the brain. New York: Oxford University Press.

Shuler MG, Krupa DJ, Nicolelis MAL (2001) Bilateral integration of whisker information in the primary somatosensory cortex of rats. $J$ Neurosci 21:5251-5261.

Shuler MG, Krupa DJ, Nicolelis MAL (2002) Integration of bilateral whisker stimuli in rats: role of the whisker barrel cortices. Cereb Cortex 12:86-97.

Sparta DR, Stamatakis AM, Phillips JL, Hovelsø N, Van Zessen R, Stuber GD (2011) Construction of implantable optical fibers for long-term optogenetic manipulation of neural circuits. Nat Protoc 7:12-23.

Storace DA, Cohen LB (2017) Measuring the olfactory bulb inputoutput transformation reveals a contribution to the perception of odorant concentration invariance. Nat Commun 8:81.

Thuerauf N, Gossler A, Lunkenheimer J, Lunkenheimer B, Maihöfner C, Bleich S, Kornhuber J, Markovic K, Reulbach U (2008) Olfactory lateralization: odor intensity but not the hedonic estimation is lateralized. Neurosci Lett 438:228-232.

Uchida N, Takahashi YK, Tanifuji M, Mori K (2000) Odor maps in the mammalian olfactory bulb: domain organization and odorant structural features. Nat Neurosci 3:1035-1043.

Ung K, Arenkiel BR (2012) Fiber-optic implantation for chronic optogenetic stimulation of brain tissue. J Vis $\operatorname{Exp}(68): 050004$.

Wilson DA (1997) Binaral interaction to the rat piriform cortex. J Neurophysiol 78:160-169.

Yan Z, Tan J, Qin C, Lu Y, Ding C, Luo M (2008) Precise circuitry links bilaterally symmetric olfactory maps. Neuron 58:613-624.

Zhou W, Chen D (2009) Binaral rivalry between the Nostrils and in the cortex. Curr Biol 19:1561-1565.

Zhou Z, Belluscio L (2012) Coding odorant concentration through activation timing between the medial and lateral olfactory bulb. Cell Rep 2:1143-1150. 